“ (C) 2017 IEEE. Personal use of this material is permitted. Permission from IEEE must be obtained for all other uses, in any current or future media, including

reprinting/republishing this material for advertising or promotional purposes, creating new collective works, for resale or redistribution to servers or lists, or reuse of any copyrighted component of this work in other works." 


\title{
Sum-Frequency Generation and Photon-Pair Creation in AlGaAs Nano-Scale Resonators
}

\author{
Giuseppe Marino ${ }^{1,2}$, Alexander S. Solntsev ${ }^{1, *}$, Lei $\mathrm{Xu}^{1,3}$, Valerio Gili ${ }^{4}$, Luca Carletti ${ }^{5}$, \\ Alexander N. Poddubny ${ }^{1,6,7}$, Daria Smirnova ${ }^{1}$, Haitao Chen ${ }^{1}$, Guoquan Zhang ${ }^{3}$, Anatoly Zayats ${ }^{2}$, \\ Costantino De Angelis ${ }^{5}$, Giuseppe Leo ${ }^{4}$, Yuri S. Kivshar ${ }^{1}$, Andrey A. Sukhorukov ${ }^{1}$, and Dragomir N. Neshev ${ }^{1}$ \\ ${ }^{1}$ Nonlinear Physics Centre, Research School of Physics and Engineering, Australian National University, Canberra ACT 2601, Australia \\ ${ }^{2}$ Department of Physics, King's College London, Strand, London WC2R $2 L S$, UK \\ ${ }^{3}$ The MOE Key Laboratory of Weak Light Nonlinear Photonics, School of Physics, Nankai University, Tianjin, China \\ ${ }^{4}$ Matériaux et Phénoménes Quantiques, Université Paris Diderot-CNRS, 10 rue Alice Domon et Lonie Duquet, F-75013 Paris, France \\ ${ }^{5}$ Dept. of Information Engineering, University of Brescia, Via Branze 38, 25123 Brescia, Italy \\ ${ }^{6}$ ITMO University, 49 Kronverksky Pr., Saint Petersburg 197101, Russia \\ ${ }^{7}$ Ioffe Physical-Technical Institute, Saint Petersburg 194021, Russia \\ "Alexander.Solntsev@anu.edu.au
}

\begin{abstract}
We demonstrate experimentally efficient sum-frequency generation in AlGaAs nanoresonators, establishing a quantum-classical correspondence with spontaneous parametric downconversion. We predict that AlGaAs nano-resonators can be utilized as high-rate sources of photon pairs with non-classical correlations.

OCIS codes: (190.2620) Harmonic generation and mixing; (270.0270) Quantum optics; (050.6624) Subwavelength structures; (130.3120) Integrated optics devices; (270.5290) Photon statistics.
\end{abstract}

Introduction: All-dielectric nonlinear nano-photonics is an emerging field enabling efficient optical interactions at sub-wavelength scales, while achieving high figures of merit due to very low losses. It was shown that AlGaAs nanodisks with quadratic nonlinear susceptibility can provide second harmonic generation (SHG) efficiency of $10^{-4}$ $[1,2]$. This record-higher efficiency opens a wide range of possible applications, including nonlinear microscopy [3] and holography. In this work, we show experimentally that the strong quadratic nonlinearity in AlGaAs nanodisks allows for efficient sum-frequency generation (SFG) with nontrivial polarization dependencies. By using the established classical-quantum analogy, we predict that these nano-resonators can facilitate efficient generation of quantum entangled photon pairs with higher than $\mathrm{kHz}$ biphoton rate and strong angular correlations.

(a)

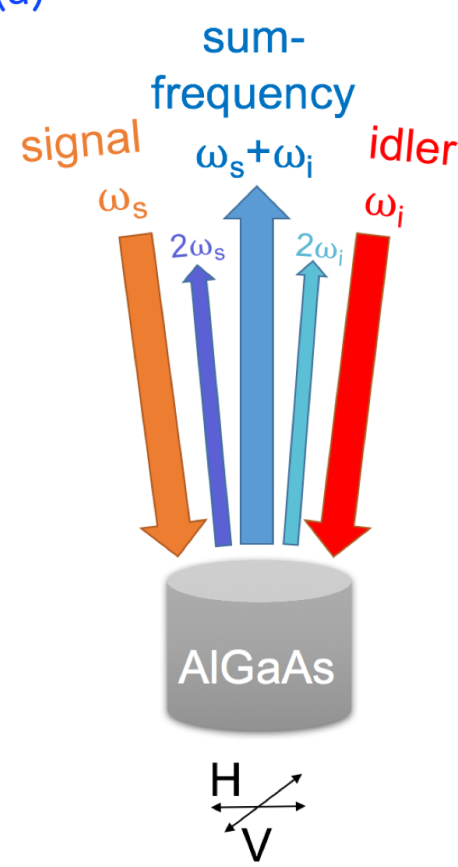

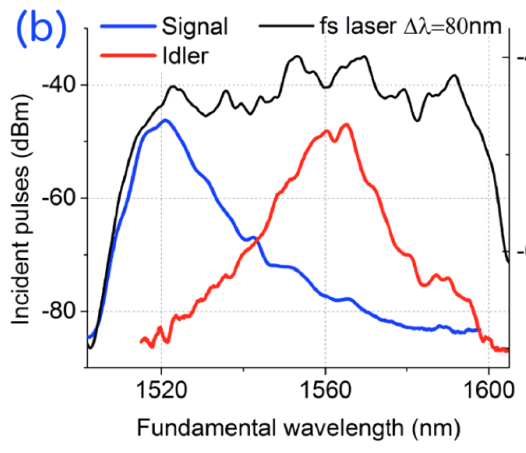

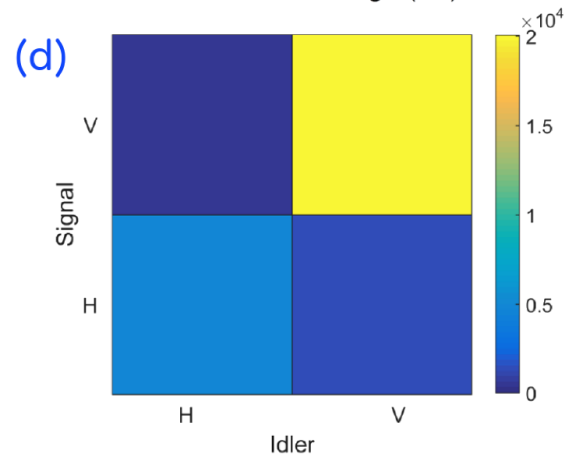

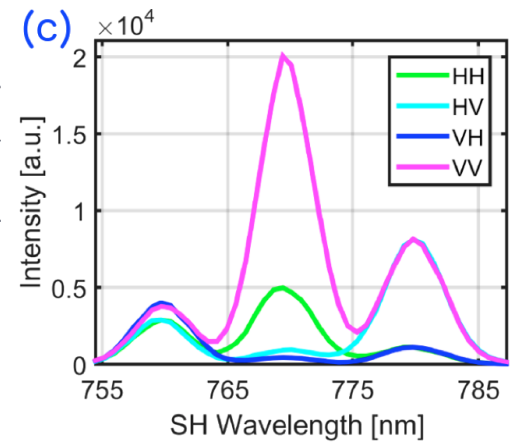

(e)

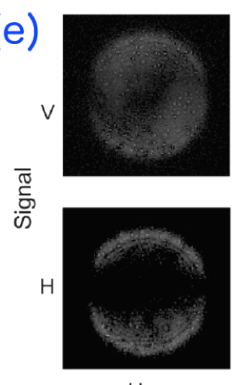

$\mathrm{H}$
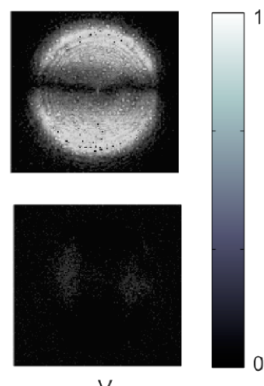

Idler

Fig. 1. Experimental observation of SFG. (a) Scheme of SFG with signal and idler beams in an [100] AlGaAs nano-resonator. The horizontal (H) and vertical (V) polarization axes are parallel to the crystalline axes of the AlGaAs lattice. (b) Signal spectrum (blue line) and idler spectrum (red line) filtered from a fs laser source (black line). (c) AlGaAs nano-resonator SFG reflection spectra at $770 \mathrm{~nm}$ and signal / idler SHG spectra at 760 $/ 780 \mathrm{~nm}$; the plot shows 4 combinations of $\mathrm{H}$ and V polarizations of signal and idler beams, respectively. (d) Color map of $\mathrm{H}$-polarized SFG at 770 $\mathrm{nm}$ for the polarization combinations shown in (c). (e) SFG directionality diagrams for the polarization combinations shown in (d). 
(a)

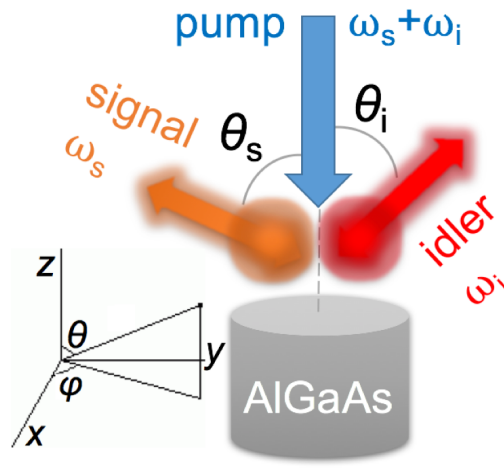

(b)

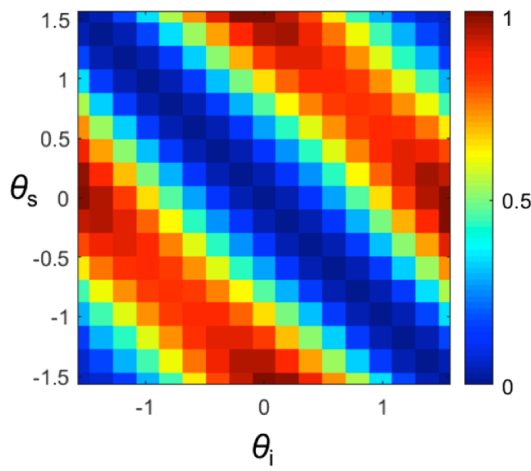

(c)

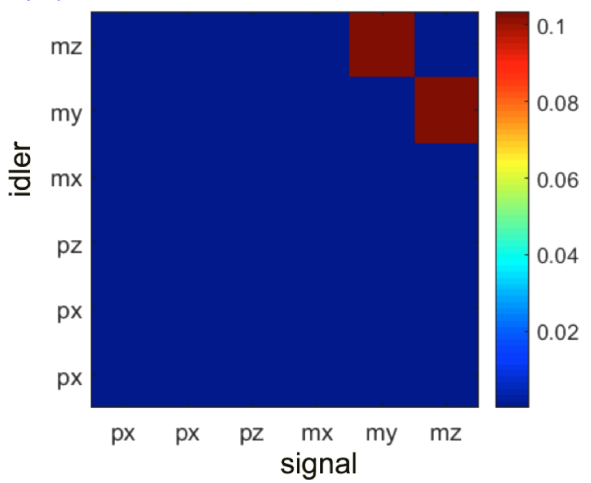

Fig. 2. (a) Photon-pair generation through SPDC from a pump beam. (b) Numerical normalized angular correlations of the signal and idler photons. (c) Numerical Cartesian dipole moments of the signal and idler photons. In all cases pump is normally incident and linear $\mathrm{x}$ (V)-polarized.

Sum-frequency generation: To measure the SFG from an [100] AlGaAs-on-oxide monolithic nano-resonator [4], as shown in Fig. 1 (a), we split a 86 fs long (bandwidth of $80 \mathrm{~nm}$ ) laser pulse into two paths: the signal arm with a central wavelength of $1520 \mathrm{~nm}$ and the idler arm with a central wavelength of $1560 \mathrm{~nm}$, see Fig. 1(b). We recombine these two beams and focus them onto a single $400 \mathrm{~nm}$-high and $420 \mathrm{~nm}$-wide AlGaAs nanodisk resonator using a $0.7 \mathrm{NA}$ objective. The polarization in each arm can be controlled independently with half-wave plates. After adjusting the optical delay between both arms such that both the signal and idler pulses arrive to the nano-resonator simultaneously, in reflection we observe three spectral peaks as a result of the nonlinear parametric wave-mixing in the nano-resonators [Fig. 1(c)]: one peak at $770 \mathrm{~nm}$ corresponding to the SFG, and two other peaks at $760 \mathrm{~nm}$ and $780 \mathrm{~nm}$, corresponding to the signal and idler SHG, respectively. We adjust the half-wave plates to scan through different combinations of $\mathrm{H}$ and $\mathrm{V}$ polarizations in the signal and idler arms, and measure the SFG with H polarization. The resulting SFG signal counts at $770 \mathrm{~nm}$ are shown in Fig. 1(d), and the corresponding SFG radiation patterns measured via a camera and a Fourier imaging system are depicted in Fig. 1(e). The highest SFG conversion efficiency measured from our resonators is over $10^{-6}$ and can be observed when the polarizations of both signal and idler arms are V-polarized, while the output polarization is horizontal. The SFG radiation patterns strongly depend on signal/idler polarization combinations. We observe good agreement between the experimental results and our COMSOL finite element method simulations.

Spontaneous parametric down-conversion: Next, we analyze the integrated generation of entangled photon pairs through spontaneous parametric down-conversion (SPDC) [5]. We formulate and employ a general quantum-classical correspondence between SPDC and SFG for arbitrary nano-photonic structures, drawing on Green function formalism [6]. The key result is that the number of photon pairs generated through SPDC is proportional to the SFG amplitude of classical signal and idler waves, propagating in the opposite direction. This allows us to predict the efficiency and correlations for the photon pairs generated by a pump beam with normal incidence on nano-resonator, by looking at the corresponding SFG picture with the reversed directions of the interacting waves [Fig. 2(a)]. For SPDC calculations we switch from polarization to angular correlations to demonstrate that an AlGaAs nano-resonator is a flexible system, able to support nontrivial three-wave mixing dependencies across different degrees of freedom. We choose polarizations of all waves to be linear along $x$ (i.e. V-polarized) by assuming polarization filtering. The photon-pair angular correlations for the pump wavelength of $770 \mathrm{~nm}$ are demonstrated in Fig. 2(b), featuring pronounced angular dependence $\theta_{\mathrm{s}}+\theta_{\mathrm{i}}= \pm \pi / 2$, where $\theta_{\mathrm{s}}$ is a signal photon reflection angle and $\theta_{\mathrm{i}}$ is an idler photon reflection angle. To understand this behavior, we perform a multipole analysis, and discover that in this case a photon-pair is generated with the entangled magnetic dipole Cartesian projections $|\mathrm{y}, \mathrm{z}>+| \mathrm{z}, \mathrm{y}>[$ Fig. 2(c)], which leads to the angular correlations shown in Fig. 2(b). These features arise due to the symmetry properties of the quadratic nonlinear susceptibility tensor for AlGaAs, which only has nonzero components for orthogonally polarized waves in threewave-mixing processes. Finally, we estimate the SPDC photon-pair rate that can be collected with a 0.7 NA objective, based on our experimental SFG efficiency measurements. We predict that a sub-wavelength AlGaAs nano-resonator can generate $10^{4}$ photon pairs per second, which is comparable with some established bulk photon pair sources and demonstrates that nonlinearity on the nanoscale can be potentially employed for quantum state generation.

\section{References}

[1] A. I. Kuznetsov et al., Science 354, 846 (2016).

[2] R. Camacho et al., Nano Lett. 16, $7191-7197$ (2016).

[3] A. Sergeyev et al., ACS Photonics 2, 687-691 (2015).

[4] V. F. Gili et al., Opt. Expr. 24, 15965-15971 (2016).

[5] A. S. Solntsev et al., Rev. Phys. (2016); doi: 10.1016/j.revip.2016.11.003

[6] A. N. Poddubny et al., Phys. Rev. Lett. 117, 123901 (2016). 